\title{
CFD Analysis of Supersonic Nozzle with Varying Divergent Profile
}

\author{
Kaviya sundar ${ }^{\# 1}$, Thanikaivel Murugan. D ${ }^{* 2}$ \\ ${ }^{\#}$ UG Degree holder, B.E. Aeronautical Engineering, Jeppiaar Engineering college, Chennai, India, \\ ${ }^{1}$ kavisun03@gmail.com \\ *Assistant Professor, Department of Aeronautical Engineering, Jeppiaar Engineering college, Chennai, India, \\ ${ }^{2}$ thanikavel.d@gmail.com
}

\begin{abstract}
The exit cross section shape of nozzle is main parameter in expansion process, and it plays the major role in mixing characteristics of jet. The study on flow characteristics of the circular nozzle have been performed widely, while that of the non-circular nozzles is not so popular. Hence, the study of flow characteristics on C-D nozzle with different geometry is analysed. C-D Nozzle with constant area ratio of 1.44 with design Mach number 1.8 is examined for the pressure of 4 and 6 atm for circular, rectangle \& square geometries and the comparison of, under expanded, perfectly expanded condition of nozzle is done. C-D nozzle with varying annular shapes for both exit and throat will produce different turbulence at the exit producing changes in the mixing characteristics of the nozzle. Three geometrical shapes circle, rectangle \& square with constant area is analysed and compared. In the present investigation, the nozzles are designed in CATIA and meshed using ICEM software. CFX solver is used in the present analysis in order to get the flow parameters, such as pressure, Mach number and total pressure, for different geometries of same area has been studied.
\end{abstract}

\section{Keyword- Flow Characteristic, CD Nozzle, Core length, Constant Area Ratio, CFX solver}

\section{INTRODUCTION}

The free shear flow driven by momentum usually introduced at the exit of the nozzle or orifice is called as jet. They exhibit a characteristic that "the ratio of width to axial distance of the nozzle is constant. The magnitude of the constant varies inversely with jet Mach number. Classification of jet is done based on Mach number, Mach number less than 0.3 represents the incompressible jets. For the Mach number equal to or greater than 0.3 is termed as compressible flow. Potential core region is the region in which the axial velocity remains constant with respect to jet exit velocity, surrounded by rapidly growing shear layer with intense turbulence. The expansion of jets are classified based on their exit pressure, when the exit pressure is less than the ambient pressure then the jet is said to be over expanded jet, which says that oblique shocks are formed at the exit of the nozzle and they are reflected as expansion waves from the boundary of the jet .If the exit pressure is equal to the ambient pressure then the jet is called as correctly expanded jets, which gives a periodic wave structure .when the exit pressure is higher when compared to ambient pressure, which gives wedge shaped expansion at the exit of the nozzle. This type of expansion is called as under expanded jets. Mixing characteristics of a jet will greatly improve the efficiency of a combustion cycle by increasing the mixing of air and fuel. The exit cross section of the nozzle plays major role in the expansion process. The study of symmetrical nozzles are performed widely when compared to non - symmetrical nozzles.

\section{LITERATURE SURVEY}

C-D nozzle is used for supersonic speeds in rockets, High speed jets. At the exit of the nozzle a jet of coherent stream is injected into the surrounding medium. The rate of mixing of supersonic jets with the surrounding medium has many applications, such as increase in combustion rate, thrust augmentation, and increase in propulsion efficiency.

S.Mahendiran and B.T.N Sridhar (2016) has investigated the flow characteristics on rectangular nozzle with differential ramp expansion at the exit in the rate of $2.5 \%, 5 \%, 7.5 \%$. The observed that rectangular nozzle with ramp expansion of $7.5 \%$ gives higher mixing rate.

N.Karthikeyan and B.T.N Sridhar (2011), examined the flow structures emanating from the triangular and circular nozzles were analysed and experimental work has been carried out . They observed that triangular shape nozzle shows higher spreading rate of jet due to the interaction of jet with vortex structure.

David Munday, Ephraim Gutmark, JunhuiLiu and K.Kaialasanath (2002), investigated the flow structure of C-D nozzle with conical sections. Experimental study shows that for contoured nozzles at off design condition, it was found that in addition to the shock diamond feature, these nozzles produce a second set of shock diamonds anchored at the nozzle throat. These together for double diamond appearance. 


\section{DESIGN OF C-D NozZLE}

Design of C-D nozzle with different geometrical shapes of constant area ratio has been done. For all the nozzles, inlet area, outlet area, throat area, convergent and divergent length are kept constant only the shapes have been changed for both throat and exit as circular, rectangle and square. The nozzles are designed for the area ratio by the following relations.

The Area Mach number relation is

$$
\frac{A}{A^{*}}=\frac{1}{M^{2}}\left[\frac{2}{\gamma+1}\left(1+\frac{\gamma-1}{2} M^{2}\right)\right]^{\frac{\gamma+1}{\gamma-1}}=1.44
$$

The Pressure ratio-Mach number relation is

$$
\frac{P_{o}}{P}=\left(1+\frac{\gamma-1}{2} M^{2}\right)^{\frac{\gamma}{\gamma-1}}=5.7803
$$

The nozzles are designed with the above parameters as constant using CATIA software and for clear understanding the geometries are given below in Table 1 .

TABLE I

COMMON PARAMETERS FOR NOZZLES

\begin{tabular}{|l|c|}
\hline \multicolumn{1}{|c|}{ PARAMETERS } & DIMENSIOI \\
\hline Inlet Diameter $\left(D_{1}\right)$ & $20 \mathrm{~mm}$ \\
\hline Inlet Area $\left(A_{1}\right)$ & $314.15 \mathrm{~mm}$ \\
\hline Throat Area $\left(A_{2}\right)$ & $78.54 \mathrm{~mm}^{2}$ \\
\hline Exit Area $\left(A_{3}\right)$ & $113.04 \mathrm{~mm}$ \\
\hline Length Of The Nozzle & $100 \mathrm{~mm}$ \\
\hline Convergent Length $\left(L_{C}\right.$ & $70 \mathrm{~mm}$ \\
\hline Divergent Length $\left(L_{D}\right)$ & $30 \mathrm{~mm}$ \\
\hline
\end{tabular}

3D projection of circle, square and rectangle is shown in the Fig $1,2 \& 3$, the figure shows that inlet for all the three models kept constant whereas shapes of the throat and exit section is changed with constant area ratio.

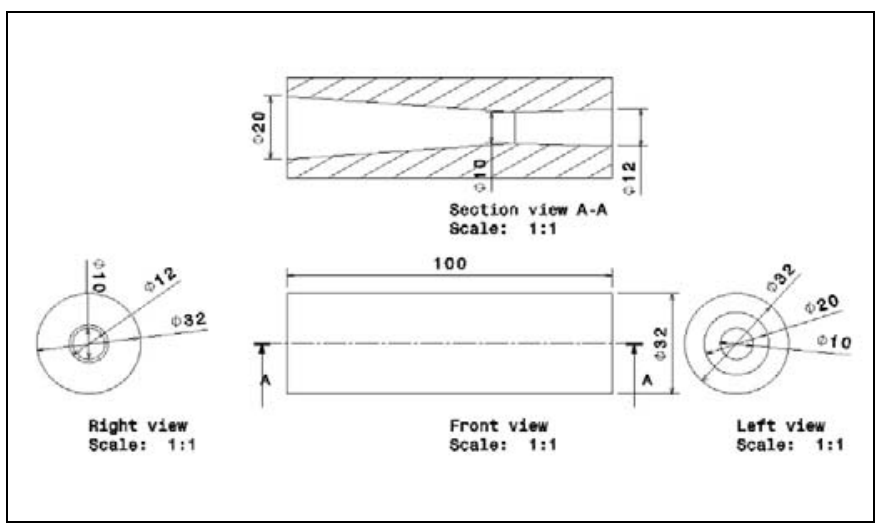

Fig. 1. Geometry model with circular shape at exit and throat

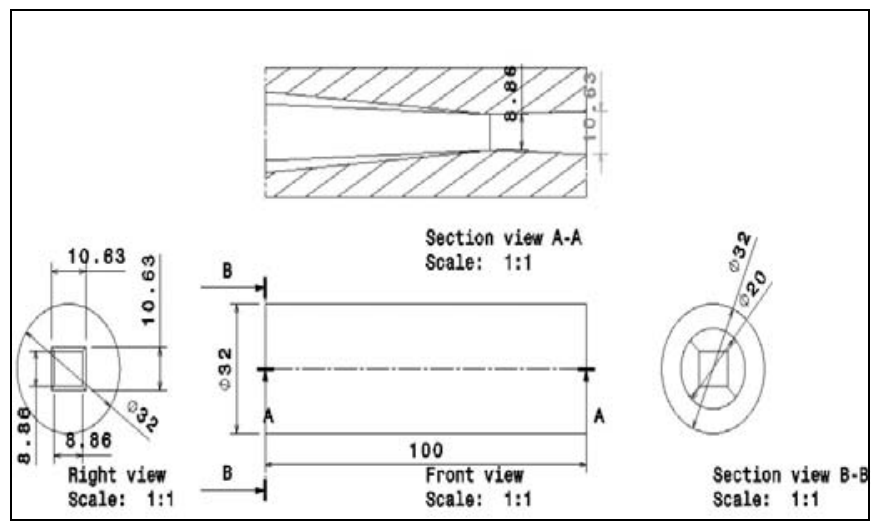

Fig. 2. Geometry model with square shape at exit and throat 


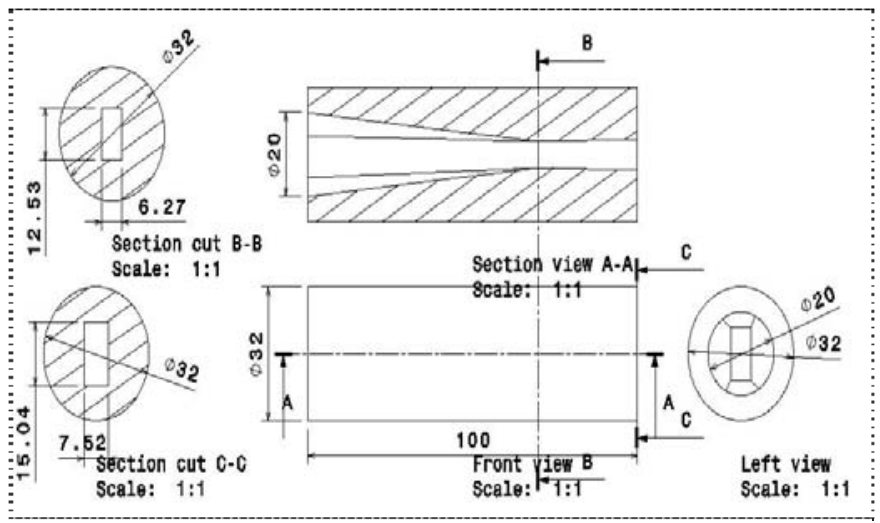

Fig. 3. Geometry model with rectangular shape at exit and throat

\section{COMPUTATIONAL ANALYSIS}

Fluid flow problems are solved by using Computational Fluid Dynamic (CFD) which uses various numerical methods as well as approximations to solve. The meshing of model is done with the ICEM Software.

In computational solutions of partial differential equations, discrete representation of geometry involved is meshing. Regular connectivity is achieved by the structured grids, they give better convergence and higher resolution. Hexahedral mesh is preferred for high accuracy and to capture the flow features. The mesh details are prescribed in Table 2 and meshed model with domain is shown in Fig. 4.

TABLE 2

Mesh Details

\begin{tabular}{|c|c|}
\hline PARAMETER & VALUE \\
\hline Element type & Hexahedron \\
\hline No. of nodes & 527276 \\
\hline No. of elements & 516480 \\
\hline
\end{tabular}

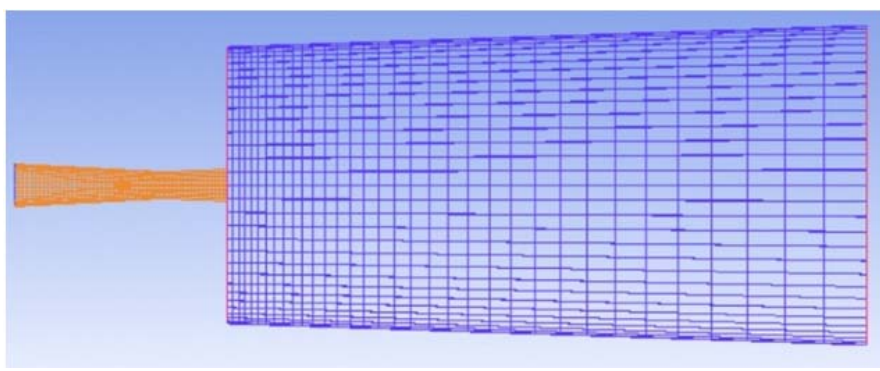

Fig. 4. Meshed Model with Domain

\section{RESUlT AND DisCUSSION}

The computational study of C-D nozzle is done for three different geometries with constant area ratios. The condition of over expanded, under expanded and perfectly expanded are analysed and compared. The simulation gives various plots of flow parameters at the exit such as Total pressure, Mach number and pressure. The nozzle jet Mach number studied is 1.8 .

\section{A. Contour Plots for Circle, Square and Rectangle Cross Section Nozzle for 4 Atm}

The Mach contour plots in Fig.5, 6 \& 7 are compared at the exit of the nozzle view. From this, circular cross section is higher (1.7797) compared to square (1.7793) and rectangle (1.775). The core length of the circle and square are same as $(70 \mathrm{~mm})$ from exit. For rectangular nozzle, the value is less $(60 \mathrm{~mm})$. 


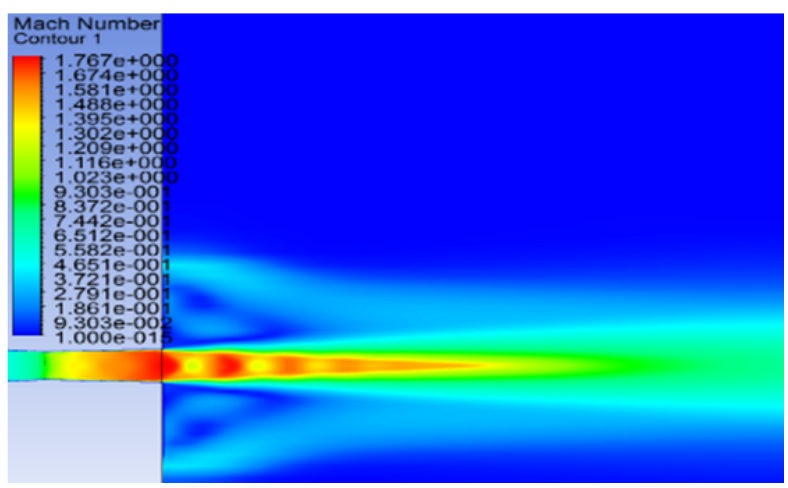

Fig. 5 Mach number contour for circle model

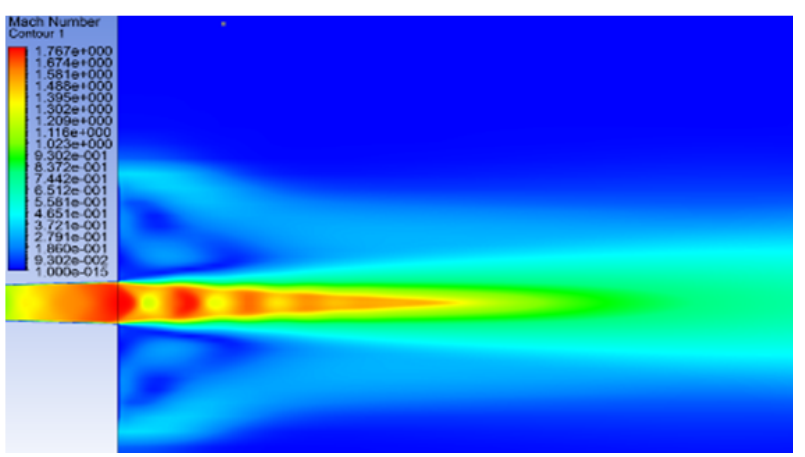

Fig. 6 Mach number contour for square model

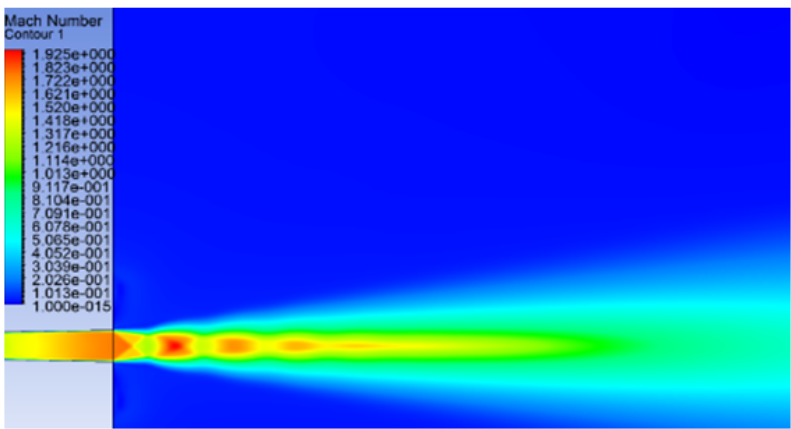

Fig. 7 Mach number contour for rectangle model

The Pressure contour plots in Fig.8, $9 \& 10$ shows that the exit pressure of the circle and square are same which gives value of 0.725 bar. The exit pressure of rectangle nozzle $(0.749)$ is higher than square and circle. The core length of circle and square geometry is same $(60 \mathrm{~mm})$ but for rectangle it is small of value $50 \mathrm{~mm}$ from the exit.

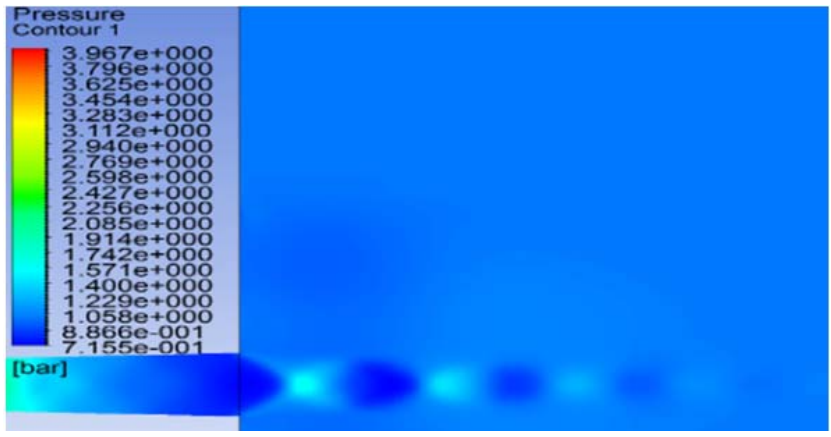

Fig. 8 Pressure contour for circle model 


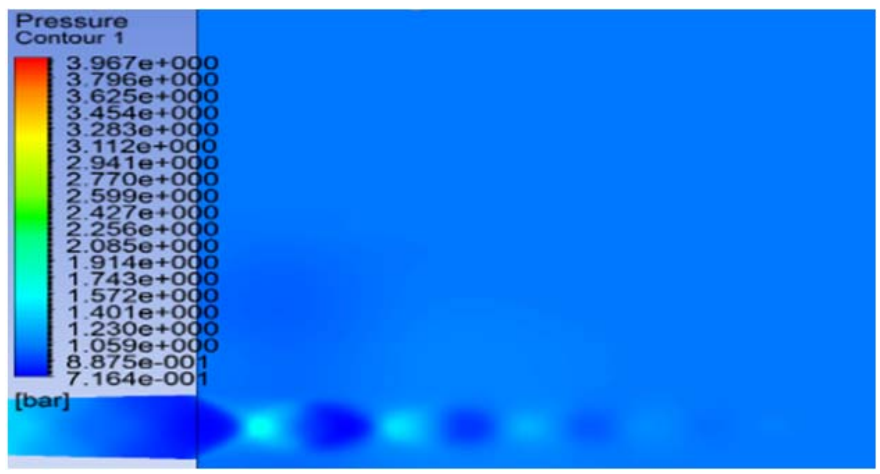

Fig. 9 Pressure contour for square model

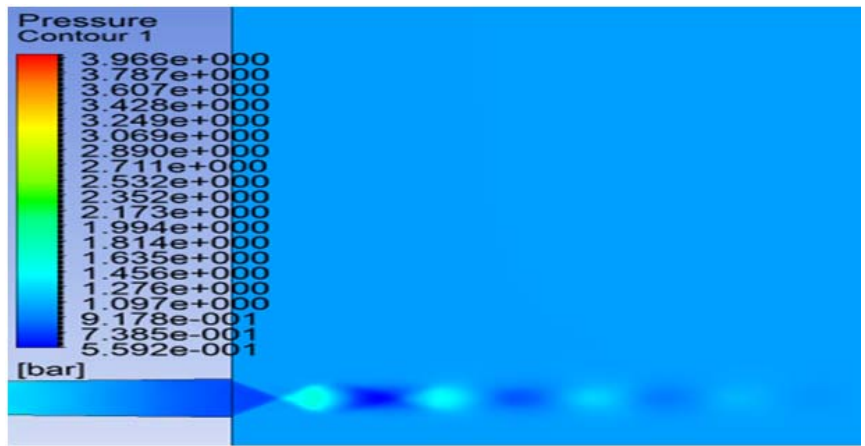

Fig. 10 Pressure contour for rectangle model

The comparison of total pressure contour in Fig.11, 12 \& 13 is shown. From this, the exit of circular and rectangle cross section is same of value 4.0431 bar. The exit total pressure of rectangle is higher (4.052) compared to both circle and square cross section. The core length of rectangular nozzle is small $(50 \mathrm{~mm})$ compared to circle and square of value $60 \mathrm{~mm}$ from the exit.

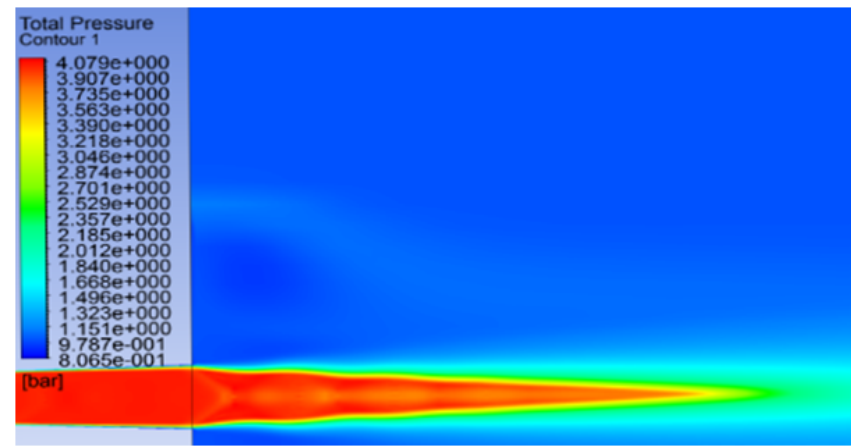

Fig. 11 Total Pressure contour for circle model

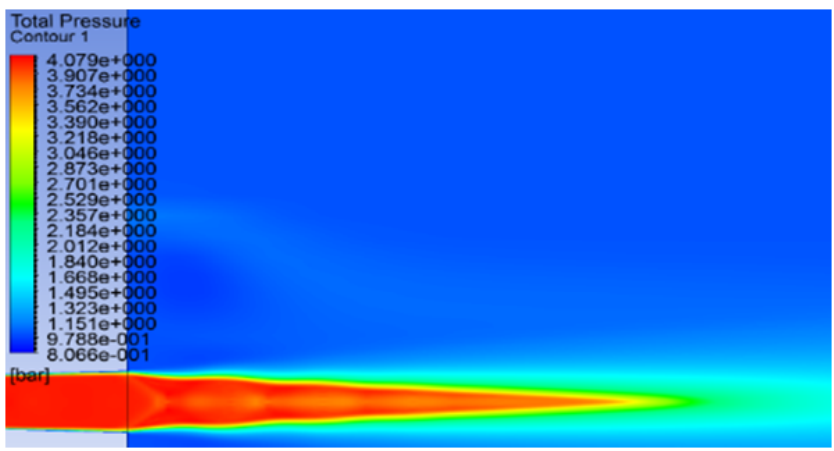

Fig. 12 Total Pressure contour for square model 


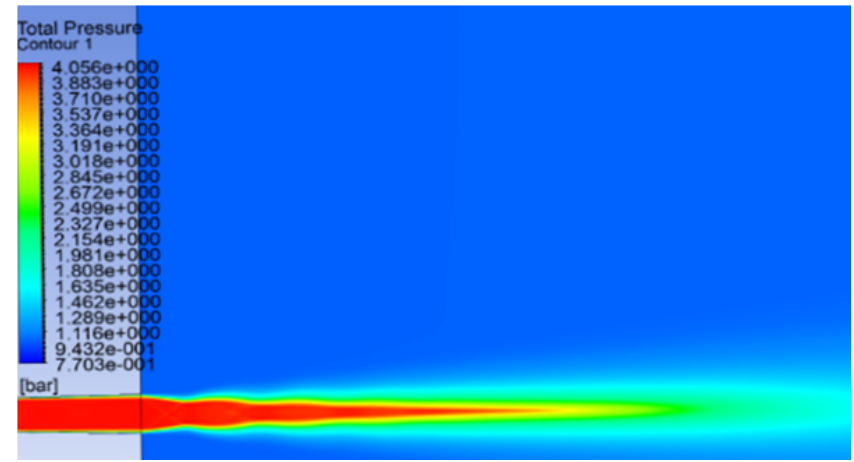

Fig. 13 Total Pressure contour for rectangle model

\section{B. Contour Plots for Circle, Square and Rectangle Cross Section Nozzle for 6 Atm}

The Mach number contour in Fig.14, 15 \& 16 are shown. From this, that the exit Mach number for the circle (1.7869) is higher compared to square (1.7817) and rectangle (1.7637) cross section. The core length of circle and rectangular nozzle is same of value $70 \mathrm{~mm}$ from the exit of the nozzle and for square is $80 \mathrm{~mm}$.

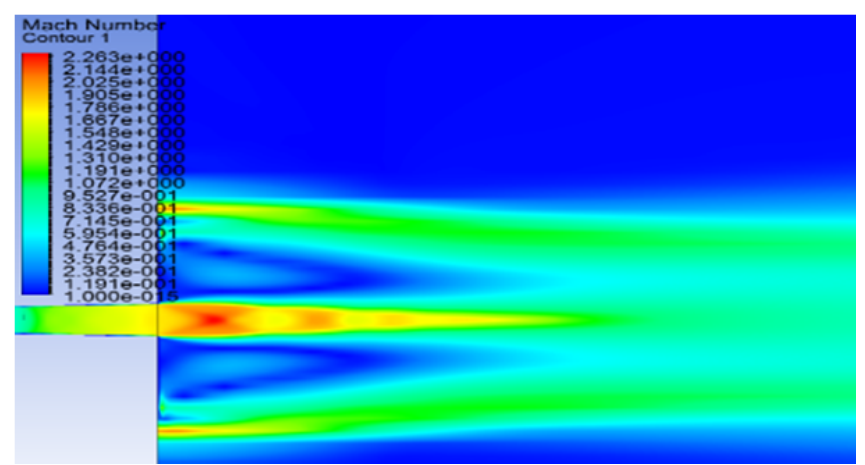

Fig. 14 Mach number contour for circle model

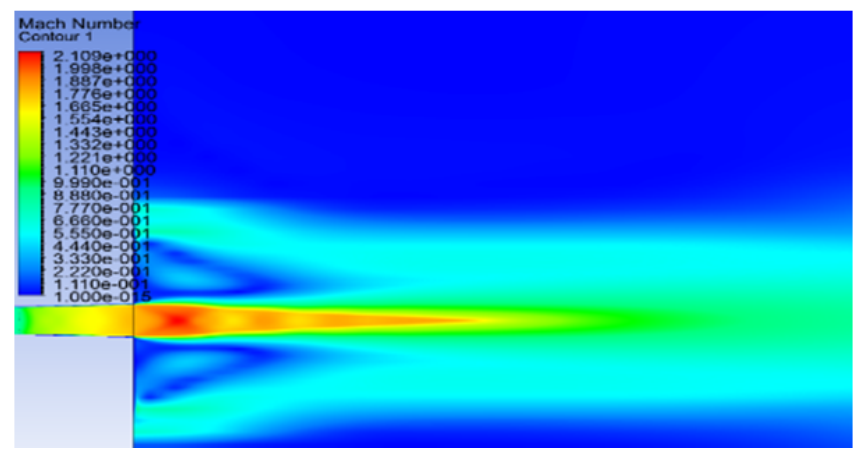

Fig. 15 Mach number contour for square model

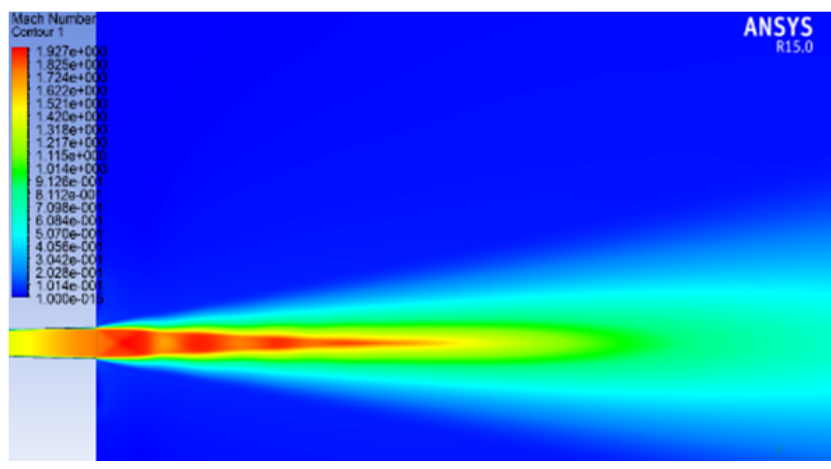

Fig. 16 Mach number contour for rectangle model 
The Pressure contour in Fig. 17, $18 \& 19$ shows that the pressure at the exit section of the circular and square cross section nozzle is same of value 1.0856 bar. The pressure at the exit of rectangular nozzle is higher than square and circle of value 1.11 bar. The core length of rectangle nozzle is small of value $60 \mathrm{~mm}$ from the exit.

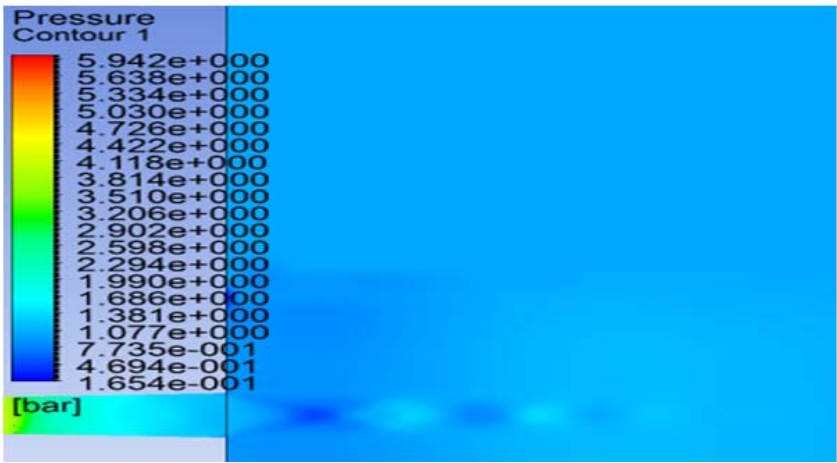

Fig. 17 Pressure contour for circle model

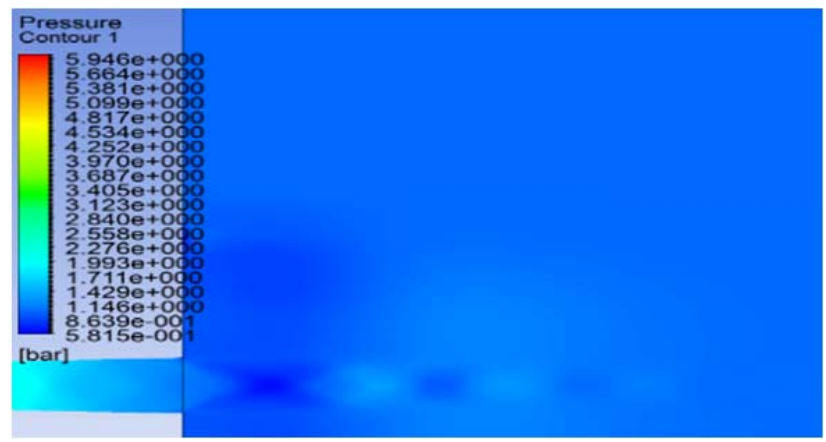

Fig. 18 Pressure contour for square model

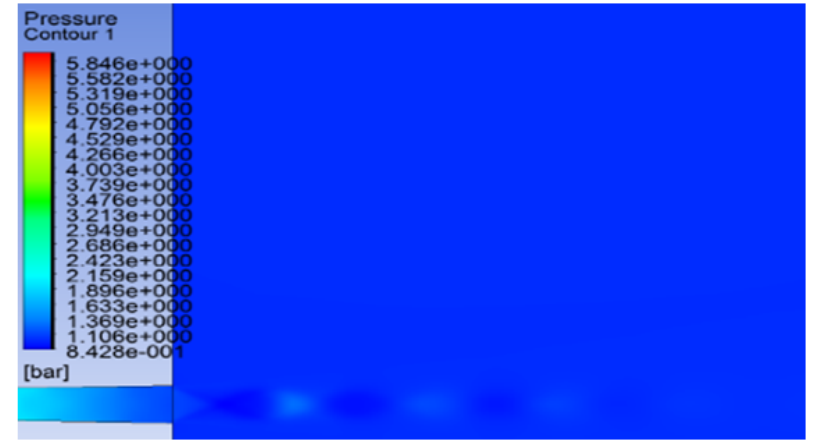

Fig. 19 Pressure contour for rectangle model

The total pressure contour in Fig.20, $21 \& 22$ shows the exit total pressure for circular and square geometry are same of value 6.66 bar. The exit total pressure of rectangle is lower of value 6.058 bar. The core length of rectangle nozzle is smaller of value $70 \mathrm{~mm}$ from the exit which shows high rate of mixing at supersonic speed.

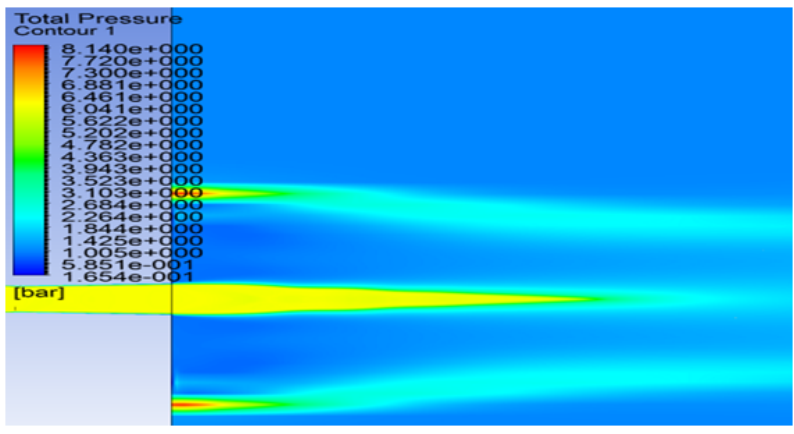

Fig. 20 Total Pressure contour for circle model 


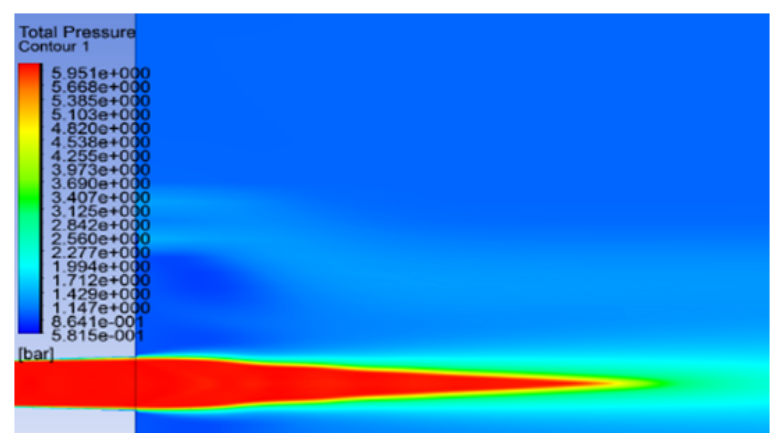

Fig. 21 Total Pressure contour for square model

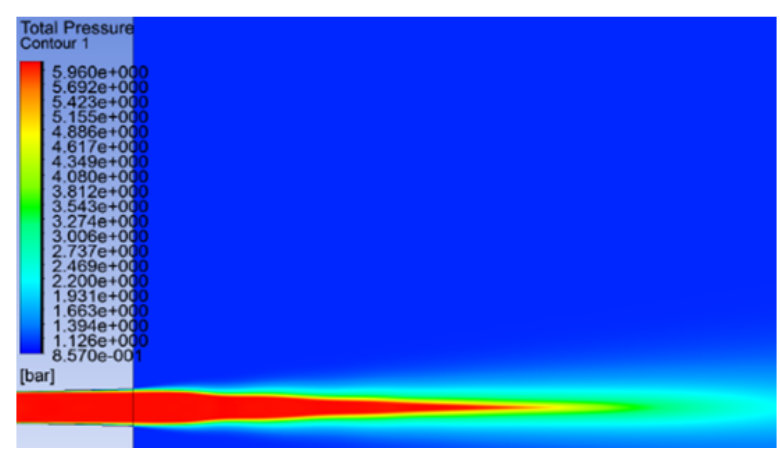

Fig. 22 Total Pressure contour for rectangle model

\section{Parameters at the Exit of the Nozzle}

CFX solver is used in the present analysis in order to get the flow parameters, such as Pressure, Mach number, and Total Pressure different geometry of same area. The parameter details obtained from the solver is shown in Table $3 \& 4$.

TABLE 3

PARAMETER DETAILS FOR 4 ATM

\begin{tabular}{|c|c|c|c|}
\hline MODELS & MACH NUMBER & PRESSURE & TOTAL PRESSURE \\
\hline CIRCLE & 1.7797 & 0.725 bar & 4.043 bar \\
\hline SQUARE & 1.7793 & 0.725 bar & 4.043 bar \\
\hline RECTANGLE & 1.759 & 0.749 bar & 4.052 bar \\
\hline
\end{tabular}

TABLE 4

PARAMETER DETAILS FOR 6 ATM

\begin{tabular}{|c|c|c|c|}
\hline MODELS & MACH NUMBER & PRESSURE & TOTAL PRESSURE \\
\hline CIRCLE & 1.7869 & $1.085 \mathrm{bar}$ & $6.066 \mathrm{bar}$ \\
\hline SQUARE & 1.78172 & $1.085 \mathrm{bar}$ & $6.066 \mathrm{bar}$ \\
\hline RECTANGLE & 1.7637 & $1.118 \mathrm{bar}$ & $6.058 \mathrm{bar}$ \\
\hline
\end{tabular}

\section{Mach Number Variation along the Jet Axis}

The graphical Fig.23 shows clear that the exit Mach number for the circle and square are same but for rectangle the curve drops first which shows the mixing characteristics of the nozzle. The core length of rectangle is small compared to the circle and square. The expansion of rectangle at the exit is higher at a distance of $10 \mathrm{~mm}$ from the exit of the nozzle. 


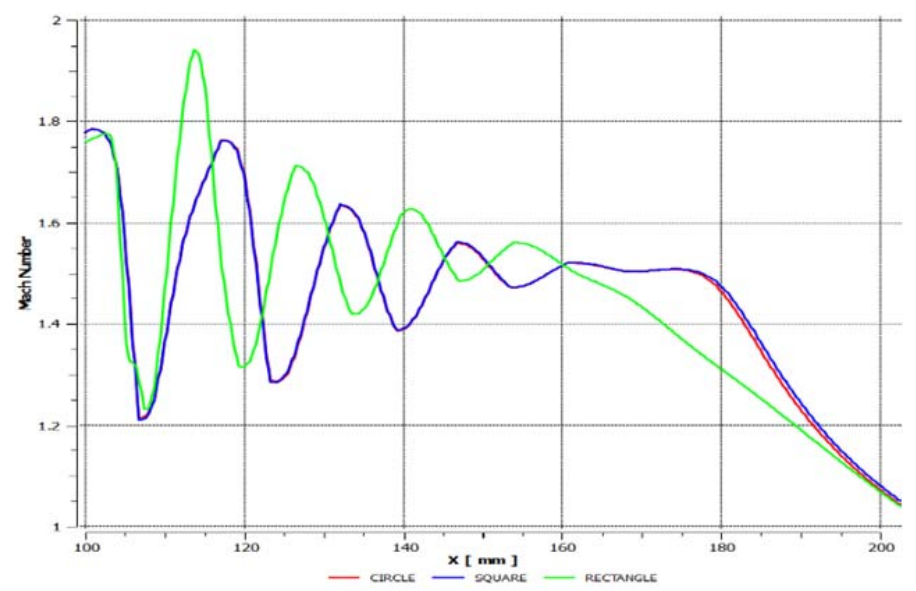

Fig. 23 Mach number comparison for $4 \mathrm{~atm}$

The Fig.24 depicts that the Mach number at the exit for the circle is higher compared to square and rectangle. The Mach number curve of circle drops firsts and the linearity is attained. Core length of rectangle is smaller when compared to the square and rectangle.

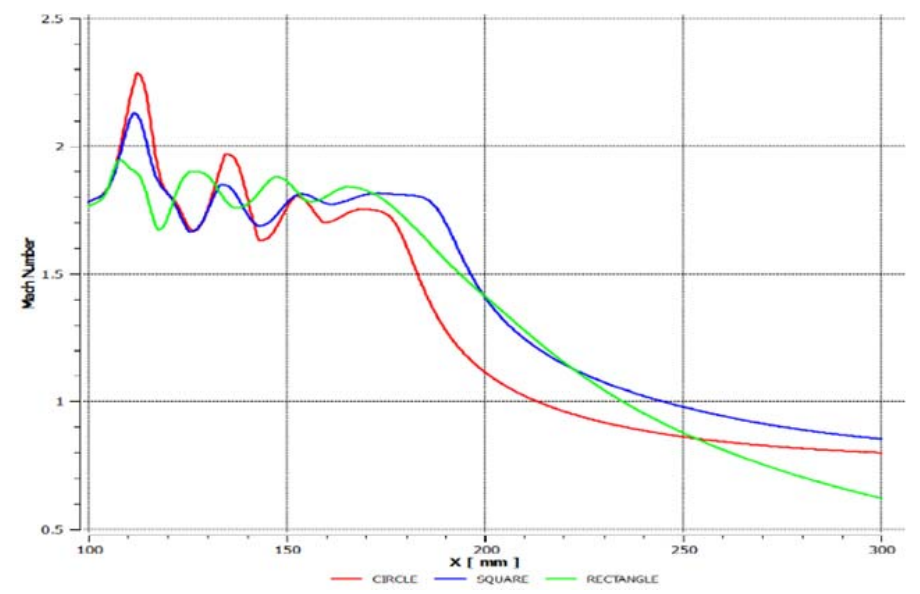

Fig. 24 Mach number comparison for 6 atm

\section{E. Pressure Variation along the Radial Direction}

The Fig. 25 shows that the circle and square have similar pressure values at the exit of the nozzle, where the rectangle nozzle gives compression and expansion process ahead of the square and circle.

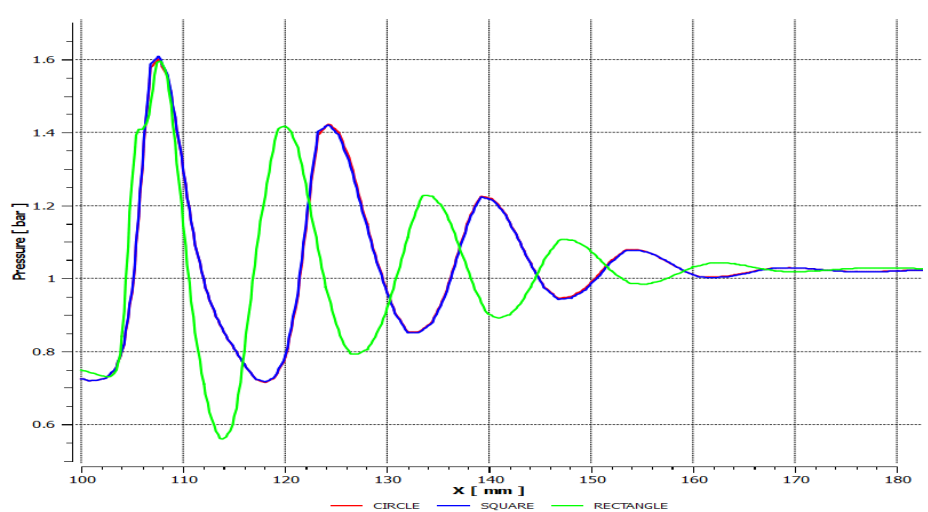

Fig. 25 Pressure comparison for $4 \mathrm{~atm}$

The Fig.26 depicts that the core length of rectangle is smaller compared to the square and circle. The value of the pressure for the rectangle area nozzle is higher at the exit. Linearity is attained by the rectangle for 6 atm pressure. 


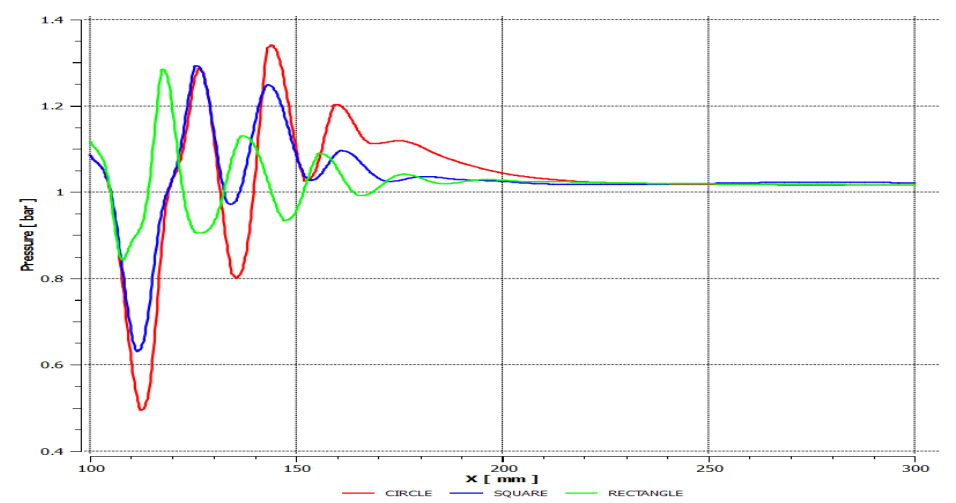

Fig. 26 Pressure comparison for 6 atm

\section{F. Total Pressure Variation along the Jet Axis}

The Fig.27 shows that the rectangle cross section nozzle will have smaller core length when compared to the square and circle. Total pressure at the exit of the circle and square is same but rectangle will give value higher total pressure compared to square and circle geometry.

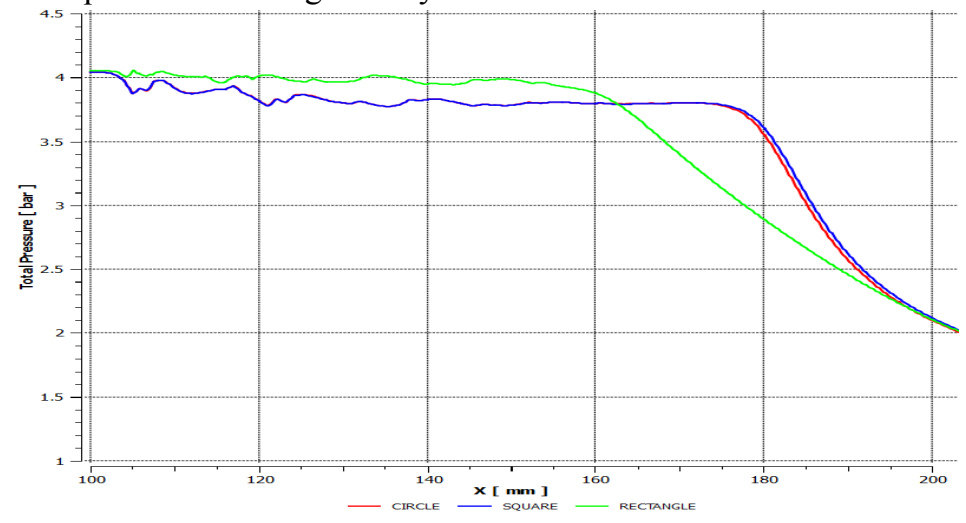

Fig. 27 Total Pressure comparison for $4 \mathrm{~atm}$

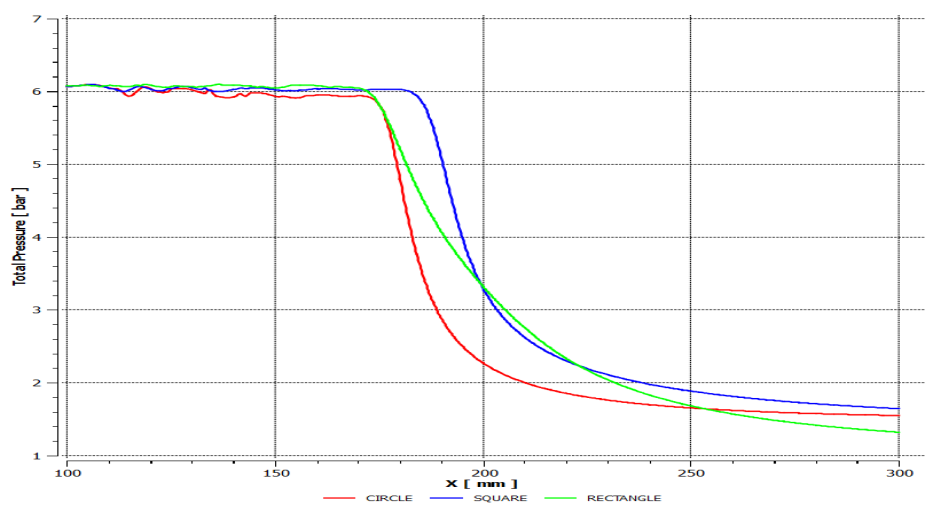

Fig. 28 Total Pressure comparison for 6atm

The above Fig. 28 shows that for 6 atm the square and circle will have different core lengths. The nozzle with circular cross section will drops first. For supersonic speed, minimum the core length maximum will be the mixing characteristics.

\section{CONCLUSION}

Based on numerical investigations of the C-D nozzle with circular, rectangle and square shapes, the result has Compared to Circle Shear strain rate of rectangular nozzle is $46 \%$ reduced and for square it is reduced to $35.5 \%$. Then Core Length of Rectangular nozzle is $6.25 \%$ reduced when compared to circle and square. From this data, it is concluded that the profile of nozzle after throat section is been changed from circle to square and then to rectangle. As the transformation of change results in reduction of shock strength cell as expands in free atmosphere. As final the rectangular profile of nozzle exit proves with less shock cell strength which is validated as best comparing to other two profiles. 


\section{ACKNOWLEDGMENT}

This work was supported by the Department of Aeronautical Engineering, Jeppiaar Engineering College. We thank our professors who provided insight and expertise that greatly assisted the research. We would also like to show our gratitude to Assistant Professor, Mr. Mahendiran for sharing his pearls of wisdom with us during the course of this research.

\section{REFERENCES}

[1] S. Mahendrian, B.T.N. Sridhar investigated on Analysis Of Jet Issuing From a Rectangular Nozzle With Differential Expansion,, National conference NCETIMS2K16, April 4th 201.

[2] N. Karthikeyan, B.T.N. Sridhar, Studies on effect of jet shapes in the coaxial supersonic jet spreading rates, International Journal of Mechanical Engineering Applications Research, Vol. 02, Issue 02; August-December 2011.

[3] David Munday and Ephraim Gutmark, "Flow structure of supersonic jets from conical C-D nozzles" member AIAA, University of Cincinnati, USA.

[4] Seung-CheolBaek, Soon-Bum Kwon, Byeong-Eun Lee, an Experimental Study of Supersonic Dual Coaxial Free Jet, KSME International Journal, Vol.17No. 12, pp.2107 2115, 2003.

[5] J. Philip Drummond, Enhancement of Mixing and Reaction in High-Speed Combustor Flow field, International Colloquium on Advanced and Analysis Of Combustion, 1997, Moscow, RUSSIA.

[6] Nicholas J.Georgiadis and Dimitri Papamoschou, Computational Investigations of High-Speed Dual-Stream Jets, 9thAIAA/CEAS Aero acoustis Conference and Exhibit, 12-14 May 2003, Hilton Head, South Carolina.

[7] D. Papamoschou and A. Zill. "Fundamental investigation of supersonic nozzle flow separation".AAIA 2004-1111, 2004.

[8] J.R. Berg, S.J. Ormiston, H.M. Soliman, "Prediction of the flow structure in a turbulent rectangular free jet." International Communications in Heat and Mass Transfer 33 (2006) 552-563.

[9] Jin sheng Cai, Her Mann Tsai, Feng Liu, "Numerical simulation of vertical flows in the near field of jets from notched circular nozzles."

[10] Steven G. Goebel and J. Craig Dutton, "Experimental study of compressible turbulent mixing layer AIAA Journal, vol.29, No.4, pp538-546, (1991).

\section{AUTHOR PROFILE}

Ms. Kaviya Sundar - Completed B.E-Aeronautical Engineering, Jeppiaar Engineering College, Chennai, Tamil Nadu, India. Her area of research interest is Aerodynamics and Supersonic Jets

Mr. Thanikaivel Murugan.D - Working as Assistant Professor, Jeppiaar Engineering College, Department of Aeronautical Engineering, Chennai, Tamil Nadu, India. His area of research interest is supersonic Jets, Aerodynamics, Aircraft Propulsion and CFD. 